\title{
SHARP ESTIMATE OF THE LAPLACIAN OF A POLYHARMONIC FUNCTION AND APPLICATIONS
}

\author{
OGNYAN IV. KOUNCHEV
}

\begin{abstract}
The classical sharp inequality of Markov estimates the values of the derivative of the polynomial of degree $n$ in the interval $[a, b]$ through the uniform norm of the polynomial in the same interval multiplied by $2 n^{2} /(b-a)$. In the present paper we provide an exact estimate for the values of the Laplacian of a polyharmonic function of degree $m$ by the uniform norm of the polyharmonic function multiplied by $2(m-1)^{2} / R^{2}(x)$ where $R(x)$ is the distance from the point $x$ to the boundary of the domain. The inequality of Markov (and the similar inequality of Bernstein about trigonometric polynomials) finds many applications in approximation theory for functions of one variable. We prove analogues to some of these results in the multivariate case.
\end{abstract}

\section{MAin RESUlts AND APPLICATIONS}

Let $\Omega$ be a bounded domain in $\mathbf{R}^{n}$. We shall denote by $H_{m}(\Omega)$ the set of polyharmonic functions $h(x)$ of order $m$, defined by the equation $\Delta^{m} h(x)=$ $0, x \in \Omega$. Here $\Delta$ is the Laplace operator in $\mathbf{R}^{n}$ given by $\sum_{j=1}^{n} \partial^{2} / \partial x_{j}^{2}$ and the polyharmonic operator $\Delta^{k}$ is defined inductively for all integers $k \geq 0$ by $\Delta^{0}=\mathrm{id}, \Delta^{1}=\Delta, \Delta^{k}=\Delta \Delta^{k-1}$.

The main result of the paper is proved in Theorem 1 and is the following inequality, announced in [1]:

$$
|\Delta h(x)| \leq \frac{2}{R^{2}(x)}(m-1)^{2}\|h\|_{C(\bar{\Omega})}
$$

for every $x \in \Omega$, and every $h(x) \in H_{m}(\Omega) \cap C(\bar{\Omega})$. Here $R(x)$ denotes the Euclidean distance from the point $x$ to the boundary $\partial \Omega$ of $\Omega$, i.e. $R(x)=$ $\inf \{|x-y|: y \in \partial \Omega\} .\|\cdot\|_{C(\bar{\Omega})}$ denotes the usual maximum modulus norm in the space $C(\bar{\Omega})$ of continuous functions on the closure $\bar{\Omega}$ of $\Omega$ given by

$$
\|f\|_{C(\bar{\Omega})}=\max _{x \in \bar{\Omega}}|f(x)| \text { for } f(x) \in C(\bar{\Omega}) .
$$

Received by the editors March 21, 1990. Presented at the 6th Southeastern Approx. Theorists Conf., March 14-16, 1991, Memphis State University, and the NATO Res. Workship on Approx. by Solutions of PDEs, Quadrature Formulae, and Related Topics, July 8-12, 1991, Hanstholm, Denmark.

1980 Mathematics Subject Classification (1985 Revision). Primary 35J30; Secondary 31B30, $41 \mathrm{~A} 17$.

Key words and phrases. Polyharmonic function, Markov inequality, Bernstein inequality, inverse theorems, Kolmogorov type inequality.

Research supported in part by Research Grant N 127 with MCPE of Bulgaria. 
This inequality may be considered as an analogue to the famous Markov inequality for polynomials $[3,6]$ :

$$
\left|P_{m}^{\prime}(t)\right| \leq m^{2}\left\|P_{m}\right\|_{C[-1,1]} \text { for every } t \in[-1,1] .
$$

Inequality (1.2) holds for every polynomial $P_{m}(t) \in \operatorname{Pol}(m)$; here and further on we denote the space of polynomials in one variable of degree $\leq m$ by $\operatorname{Pol}(m)$.

In Theorem 2 we prove a more general sharp estimate for the powers of the Laplacian, $\Delta^{k} h(x), k=1,2, \ldots, m-1$, for polyharmonic functions $h(x) \in H_{m}(\Omega) \cap C(\bar{\Omega})$. To make the things clearer and since inequality (1.1) plays an important role in the applications, we give in Theorem 1 a separate proof for the case $k=1$.

The proof is based on a proper representation of the polyharmonic functions in the ball. This representation arises from the classical Almansi representation formula.

Following the classical applications of the inequalities of Markov and Bernstein [6], in Theorem 3 we prove an inverse theorem of Bernstein type for the approximation of continuous functions through polyharmonic. As an illustration we formulate a corollary concerning approximation through polynomials of many variables showing the importance of the degree of polyharmonicity of a polynomial.

Another application is the estimate of the modulus of the intermediate derivatives of an arbitrary function proved in Theorem 4.

\section{Preliminaries}

Let us briefly introduce some necessary notions and notations and state some well-known results.

Here we fix a point $x_{0} \in \mathbf{R}^{n}$ and a positive number $R$.

1. Let us denote by $B=B\left(x_{0} ; R\right)$ the open ball in $\mathbf{R}^{n}$ with centre $x_{0}$ and radius $R$, and by $S=S\left(x_{0} ; R\right)$ the sphere which is the boundary $\partial B$ of $B$.

We recall the classical Almansi representation formula (cf. [2, 4]):

Every $h(x) \in H_{m}(B) \cap C(\bar{B})$ and $B=B\left(x_{0} ; R\right)$ may be written as

$$
h(x)=\sum_{k=0}^{m-1} r^{2 k} h_{k}(x), \quad r=\left|x-x_{0}\right|,
$$

where $h_{k}(x), k=0,1, \ldots, m-1$, are harmonic functions, i.e. $h_{k}(x) \in$ $H_{1}(B) \cap C(\bar{B})$.

2. Let us recall the simplest facts about the Dirichlet problem in the ball $B=B\left(x_{0} ; R\right)$ [5]. For every function $f(x) \in C(S), S=S\left(x_{0} ; R\right)$, the Poisson kernel

$$
K_{n}(x, y)=\left(R^{2}-\left|x-x_{0}\right|^{2}\right)|x-y|^{-n}\left(\sigma_{n} R\right)^{-1}
$$

gives the function $h_{f}(x)$ through the following formula:

$$
h_{f}(x)=\int_{S} K_{n}(x-y) f(y) d \sigma(y), \quad \text { for } x \in B\left(x_{0} ; R\right) ;
$$

here $d \sigma(y)$ is the surface element of the sphere $S$, and the constant $\sigma_{n}$ is the area of the unit sphere in $\mathbf{R}^{n}$. 
The function $h_{f}(x)$ satisfies the Laplace equation

$$
\Delta h_{f}(x)=0 \text { for } x \in B\left(x_{0} ; R\right)
$$

and the Dirichlet boundary conditions

$$
h_{f}(x)=f(x) \text { for } x \in S\left(x_{0} ; R\right) .
$$

3. In what follows we shall consider $m$ concentric spheres $S_{j}=S\left(x_{0} ; t_{j}\right)$, $0 \leq t_{m} \leq \cdots \leq t_{1} \leq R$. We shall denote by $K_{j}$ the Poisson operator given by (2.2) for the sphere $S=S_{j}$, i.e. we set $K_{j}[f](x)=h_{f_{\mid S_{j}}}(x)$ for every $x \in$ $B\left(x_{0} ; t_{j}\right)$.

Let $P_{q}(t) \in \operatorname{Pol}(q)$. Then it is not difficult to check that $P_{q}\left(r^{2}\right)$, where $r=$ $\left|x-x_{0}\right|$, is a polyharmonic function of order $q+1$, i.e. $P_{q}\left(r^{2}\right) \in H_{q+1}(\Omega) \cap C(\bar{\Omega})$ (cf. [2]).

If we put $f(x)=P_{q}\left(r^{2}\right)$ we get $K_{j}[f](x)=P_{q}\left(t_{j}^{2}\right)$ since $r=t_{j}$ for $x \in S_{j}$.

In general, for every function $f(x) \in C\left(\bar{B}\left(x_{0} ; R\right)\right)$ such that $f(x)=f(r)$ we have $K_{j}[f](x)=f\left(t_{j}\right)$ for every $x \in B\left(x_{0} ; R\right)$.

When $t_{m} \rightarrow 0$ we get $K_{m}[f]\left(x_{0}\right) \rightarrow f\left(x_{0}\right)$. So when $t_{m}=0$ we shall write $K_{m}[f]\left(x_{0}\right)=f\left(x_{0}\right)$. In general, the case $t_{m}=0$ will be considered as a limiting with $t_{m} \rightarrow 0$.

4. Denote by $\Pi(t)$ the product $\prod_{k=1}^{m}\left(t-t_{k}^{2}\right)$ and introduce the polynomials $P_{j}(t)=\Pi(t)\left(t-t_{j}^{2}\right)^{-1}\left(\Pi^{\prime}\left(t_{j}^{2}\right)\right)^{-1}$, for $j=1,2, \ldots, m$.

These polynomials have an easy-to-check property which is the following:

$$
\begin{array}{ll}
P_{j}\left(t_{k}^{2}\right)=0 & \text { for } k \neq j, \\
P_{j}\left(t_{j}^{2}\right)=1 & \text { for } j, k=1,2, \ldots, m .
\end{array}
$$

\section{Estimation OF THE LAPLACian}

We shall need the following representation result:

Lemma 1. In the notations introduced above, for every function $h \in H_{m}(B) \cap$ $C(\bar{B})$ in the ball $B=B\left(x_{0} ; R\right)$, the equality

$$
h(x)=\sum_{j=1}^{m} P_{j}\left(r^{2}\right) K_{j}[h](x)
$$

holds, where $r=\left|x-x_{0}\right|$ and $r \leq t_{m}$.

Proof. In view of the Almansi representation formula (2.1) and the linearity of the operators $K_{j}$, it suffices to prove formula (3.1) in the case of functions of the type $h(x)=r^{2 k} v(x), k=0,1, \ldots, m-1$, where $v(x)$ is an arbitrary harmonic function in $B\left(x_{0} ; R\right)$. A direct substitution of such $h(x)$ in (3.1) gives

$$
r^{2 k} v(x)=\sum_{j=1}^{m} P_{j}\left(r^{2}\right) t_{j}^{2 k} v(x)
$$

for $k=0,1, \ldots, m-1$, by the properties of the operators $K_{j}$. So it remains to prove the identities

$$
t^{k}=\sum_{j=1}^{m} P_{j}(t) t_{j}^{2 k}
$$

for $k=0,1, \ldots, m-1$. 
On the right-hand side we have a polynomial of degree less than or equal to $m-1$ as well as on the left side. According to the property (2.3) of the polynomials $P_{j}(t)$, identity (3.2) is true for $m$ points, $t=t_{1}^{2}, t_{2}^{2}, \ldots, t_{m}^{2}$. This implies that (3.2) is true for every $t$.

The proof of (3.1) is finished. Q.E.D.

Our main idea for proving the inequality $(1.1)$ is based on a localization, so we shall be interested mainly in the values $\Delta h\left(x_{0}\right)$.

Proposition 1. For every polyharmonic function $h(x) \in H_{m}(B) \cap C(\bar{B})$ in the ball $B=B\left(x_{0} ; R\right)$, the equality

$$
\Delta h\left(x_{0}\right)=2 n \sum_{j=1}^{m} P_{j}^{\prime}(0) K_{j}[h]\left(x_{0}\right)
$$

holds.

Proof. Formula (3.1), together with the fact that $K_{j}[h](x), j=1, \ldots, m$, are harmonic functions, gives

$$
\Delta h(x)=\sum_{j=1}^{m}\left[\Delta P_{j}\left(r^{2}\right) K_{j}[h](x)+2\left\langle\operatorname{grad}\left(P_{j}\left(r^{2}\right)\right), \operatorname{grad}\left(K_{j}[h](x)\right\rangle\right]\right.
$$

for $r=\left|x-x_{0}\right| \leq t_{m}$; here $\langle$,$\rangle denotes the scalar product in \mathbf{R}^{n}$.

To calculate that the second term in the sum is zero, note that

$$
\operatorname{grad}\left(P_{j}\left(r^{2}\right)\right)=2 \cdot\left\langle x-x_{0}, P_{j}^{\prime}\left(r^{2}\right)\right\rangle .
$$

This is evidently zero for $x=x_{0}$.

To calculate $\Delta\left(P_{j}\left(r^{2}\right)\right)$, recall that $\Delta=\partial^{2} / \partial r^{2}+(n-1) r^{-1} \partial / \partial r$ on functions depending only on $r$ [5]. This gives

$$
\Delta\left(P_{j}\left(r^{2}\right)\right)=2 n P_{j}^{\prime}\left(r^{2}\right)+4 r^{2} P_{j}^{\prime \prime}\left(r^{2}\right)
$$

which implies that $\Delta\left(P_{j}\left(r^{2}\right)\right)_{\mid x=x_{0}}=2 n P_{j}^{\prime}(0)$.

This completes the proof of (3.3). Q.E.D.

Formula (3.3) may be considered as an analogue to the classical M. Riesz formula for trigonometric polynomials [7].

We shall use the notation sign $(x)=x /|x|$ for $x \neq 0$.

An important feature of the proof of (1.1) is the determination of the sign of $P_{j}^{\prime}(0)$.

Proposition 2. For the signs $\operatorname{sign}\left(P_{j}^{\prime}(0)\right)$ we have

$$
\operatorname{sign}\left(P_{j}^{\prime}(0)\right)=-\operatorname{sign}\left(P_{j+1}^{\prime}(0)\right)
$$

for $j=1,2, \ldots, m-1$, i.e. they are alternating in $j$.

Proof. We shall see that the alternation (3.5) is in fact true for $\operatorname{sign}\left(P_{j}^{\prime}(t)\right)$ for $t \leq t_{m}^{2}$.

We have

$$
P_{j}^{\prime}(t)=\left(\Pi^{\prime}\left(t_{j}^{2}\right)\right)^{-1} \cdot \sum_{\substack{k=1 \\ k \neq j}}^{m} \Pi(t)\left(t-t_{j}^{2}\right)^{-1}\left(t-t_{k}^{2}\right)^{-1}
$$


Every summand $\Pi(t)\left(t-t_{j}^{2}\right)^{-1}\left(t-t_{k}^{2}\right)^{-1}$ in the sum has exactly $m-2$ multipliers with sign -1 , and consequently,

$$
\operatorname{sign}\left(\Pi(t)\left(t-t_{j}^{2}\right)^{-1}\left(t-t_{k}^{2}\right)^{-1}\right)=(-1)^{m-2} .
$$

On the other hand it is easy to see that

$$
\operatorname{sign}\left(\Pi^{\prime}\left(t_{j}^{2}\right)\right)=(-1)^{m-j} \text {. }
$$

Finally, we get that

$$
\operatorname{sign}\left(P_{j}^{\prime}(t)\right)=(-1)^{j+2} \text { for } t \leq t_{m}^{2}
$$

and particularly when $t=0$. Q.E.D.

Before starting the proof of the main result let us recall some basic properties of the Chebyshev polynomials $[3,6]$. They are given by

$$
\begin{aligned}
T_{n}(t) & =\cos (n \arccos (t)) \\
& =(1 / 2)\left[\left(t+\left(t^{2}-1\right)^{1 / 2}\right)^{n}+\left(t-\left(t^{2}-1\right)^{1 / 2}\right)^{n}\right],
\end{aligned}
$$

for $t \in[-1,1], n=0,1, \ldots$.

For $t=x_{k}=\cos (k \pi / n), k=0,1, \ldots, n$, the polynomial $T_{n}(t)$ alternatively takes the values $+1,-1$.

Another property which will be used is that the numbers $T_{n}^{(k)}(1)$ and $T_{n}^{(k)}(-1)$ have the same absolute value:

$$
T_{n}^{(k)}(1)=n^{2}\left(n^{2}-1^{2}\right) \cdots\left(n^{2}-(k-1)^{2}\right)((2 k-1) ! !)^{-1}=\left|T_{n}^{(k)}(-1)\right|
$$

for the derivatives of order $k=1,2, \ldots, n$; here we use the notation $n ! !=$ $n(n-2)(n-4) \ldots$.

Now we may prove

Theorem 1. Let in the bounded domain $\Omega \subseteq \mathbf{R}^{n}$ the polyharmonic function $h(x) \in H_{m}(\Omega) \cap C(\bar{\Omega})$. If $R(x)$ is the distance from the point $x$ to the boundary $\partial \Omega$, then the sharp estimate (1.1) is true for every point $x \in \Omega$.

Proof. Consider the point $x=x_{0} \in \Omega$.

In the ball $B=B\left(x_{0} ; R_{1}\right), R_{1}=R\left(x_{0}\right)$, we have the representation (3.3) for every family of concentric spheres $S_{j}=S\left(x_{0} ; t_{j}\right)$, for $j=1,2, \ldots, m$, and $0 \leq t_{m} \leq \cdots \leq t_{1} \leq R_{1}$. We put $R=R_{1}^{2}$.

Now we introduce the following transform of the Chebyshev polynomial $T_{m}(t)$ by substituting $t=(y-R / 2) /(R / 2), L_{m}(y)=T_{m}(t)$ for $y \in[0, R]$.

Specify further the values $t_{k}, k=1,2, \ldots, m$, by putting $\left(t_{k}^{2}-R / 2\right) /(R / 2)$ $=x_{k-1}=\cos ((k-1) \pi /(m-1))$, i.e.

$$
t_{k}=+[R / 2+(R / 2) \cos (k \pi /(m-1))]^{1 / 2} .
$$

Note that $L_{m-1}\left(t_{k+1}^{2}\right)=T_{m-1}\left(x_{k}\right)$ for $k=0,1, \ldots, m-1$, which takes alternatively the values $+1,-1$.

Let us now substitute the polyharmonic function of order $m, L(x)=$ $L_{m-1}\left(r^{2}\right), r=\left|x-x_{0}\right|$, in (3.3).

On the left-hand side, as in Proposition 1, we get

$$
\Delta L\left(x_{0}\right)=2 n L_{m-1}^{\prime}(0) \text {. }
$$


On the right-hand side we have

$$
K_{j}[L(\cdot)]\left(x_{0}\right)=L_{m-1}\left(t_{j}^{2}\right),
$$

the last being alternatively $+1,-1$ for $j=1,2, \ldots, m$. Thus, Proposition 2 provides that

$$
2 n L_{m-1}^{\prime}(0)=2 n \sum_{j=1}^{m} P_{j}^{\prime}(0) L_{m-1}\left(t_{j}^{2}\right)=2 n \varepsilon \sum_{j=1}^{m}\left|P_{j}^{\prime}(0)\right|,
$$

or

$$
L_{m-1}^{\prime}(0)=\varepsilon \sum_{j=1}^{m}\left|P_{j}^{\prime}(0)\right|
$$

where $\varepsilon=\operatorname{sign}\left[P_{m}^{\prime}(0)\right] \cdot \operatorname{sign}\left[L_{m-1}\left(t_{m}^{2}\right)\right]=\operatorname{sign}\left[P_{1}^{\prime}(0)\right] \cdot \operatorname{sign}\left[L_{m-1}(0)\right]$.

Hence for general $h(x) \in H_{m}(\Omega) \cap C(\bar{\Omega})$ we get by (3.3) the inequality

$$
\begin{aligned}
\mid \Delta h\left(x_{0}\right) & \leq \sum_{j=1}^{m}\left|P_{j}^{\prime}(0)\right|\left|K_{j}[h]\left(x_{0}\right)\right| \\
& \leq\left(\sum_{j=1}^{m}\left|P_{j}^{\prime}(0)\right|\right)\|h\|_{C(\bar{\Omega})}=\left|L_{m-1}^{\prime}(0)\right| \cdot\|h\|_{C(\bar{\Omega})} .
\end{aligned}
$$

A direct calculation shows that

$$
\begin{aligned}
L_{m-1}^{\prime} & =-(2 / R)(m-1)\left(t-t^{2}\right)^{-1 / 2} \sin ((m-1) \arccos (t)) \\
& =(2 / R) T_{m-1}^{\prime}(t),
\end{aligned}
$$

where $t=(y-R / 2) /(R / 2)$.

According to (3.7) we finally get

$$
\left|L_{m-1}^{\prime}(0)\right|=(2 / R)(m-1)^{2} \text {. }
$$

This finishes the proof of (1.1).

The sharpness of the estimate for every point $x_{0}$ follows through (3.9) applied to the function $L(x)$ defined above. Q.E.D.

\section{ESTIMATION OF THE POWERS OF THE LAPLACIAN}

We shall prove a generalization to Theorem 1 for estimation of the powers of the Laplacian.

It is based on the following representation, generalizing Proposition 1:

Proposition 3. In the notations introduced above, for every polyharmonic function $h(x) \in H_{m}(B) \cap C(\bar{B})$ in the ball $B=B\left(x_{0} ; R\right)$, the following formula holds for every integer $p \geq 0$ :

$$
\Delta^{p} h\left(x_{0}\right)=C \sum_{j=1}^{m} P_{j}^{(p)}(0) K_{j}[h]\left(x_{0}\right)
$$

here the constant $C=l_{p, 2 p}^{n}$ and the constants $l_{p, k}^{n}$ are defined below in (4.4).

Before proving this statement we need some preparatory results. 
We shall use the following formulas (cf. [2]):

$$
\Delta^{p}(u v)=\sum_{k+l+|q|=p} \frac{2^{|q|} p !}{k ! l ! q !}\left(D^{q} \Delta^{k} u\right)\left(D^{q} \Delta^{l} v\right)
$$

holding for every two functions $u, v \in C^{(2 p)}(\Omega)$ in some domain $\Omega \in \mathbf{R}^{n}$; here we denote, as usual the multi-index $q=\left(q_{1}, q_{2}, \ldots, q_{n}\right)$, with integers $q_{i} \geq 0$, $i=1,2, \ldots, n$. Another usual notations for multi-indices are

$$
|q|=q_{1}+q_{2}+\cdots+q_{n}, \quad q !=q_{1} ! q_{2} ! \cdots q_{n} !,
$$

the differentiation $D^{q}$ is defined as

$$
D^{q}=\frac{\partial^{|q|}}{\partial x^{q_{1}} \cdots \partial x^{q_{n}}} .
$$

Another useful formula is [2]:

$$
\Delta^{p}\left(r^{k}\right)=l_{p, k}^{n} \cdot r^{k-2 p}, \quad r=\left|x-x_{0}\right|,
$$

where the constants $l_{p, k}^{n}$ are given by

$$
l_{p, k}^{n}=((k ! !) /(k-2 p) ! !) \cdot((k+n-1) ! ! /(k+n-2 p-2) ! !) .
$$

Next we prove some necessary propositions.

Proposition 4. The following equality is true for every polynomial $Q_{s} \in \operatorname{Pol}(s)$, and every integer $p \geq 1$ :

and

$$
\Delta^{p} Q_{s}\left(r^{2}\right)_{\mid r=0}=l_{p, 2 p}^{n} Q_{s}^{(p)}(0), \quad r=\left|x-x_{0}\right|,
$$

for a polynomial $Q_{m-p} \in \operatorname{Pol}(m-p)$.

$$
\Delta^{p} Q_{m}\left(r^{2}\right)=Q_{m-p}\left(r^{2}\right)
$$

The proof follows directly from (4.3).

Proposition 5. Let the polyharmonic function $u(x)=Q_{s}\left(r^{2}\right)$ be given by a polynomial $Q_{s} \in \operatorname{Pol}(s)$, and the function $v(x)$ be harmonic, i.e. $v(x) \in H_{1}(\Omega)$. Then the following formula is true.

$$
\Delta^{p}(u(x) v(x))_{\mid r=0}=\Delta^{p}(u(x))_{\mid r=0} v\left(x_{0}\right)
$$

for every $x \in \Omega$; here $r=\left|x-x_{0}\right|$.

Proof. Since $\Delta v(x)=0, x \in \Omega$, formula (4.2) is reduced to

$$
\begin{aligned}
\Delta^{p}(u v) & =\sum_{k+|q|=p} \frac{2^{|q|} p !}{k ! q !}\left(D^{q} \Delta^{k} u\right)\left(D^{q} v\right) \\
& \left.=\sum_{k=0}^{p} \frac{2^{|q|} p !}{k !} \sum_{|q|=p-k} \frac{1}{q !}\left(D^{q} \Delta^{k} u\right) D^{q} v\right),
\end{aligned}
$$

so we must prove that all internal sums are zero for $0 \leq k \leq p-1$.

We shall use the formula

$$
\begin{aligned}
\left(r^{2}\right)^{s} & =\left|x-x_{0}\right|^{2 s} \\
& =\left[\left(x_{1}-x_{01}\right)^{2}+\left(x_{2}-x_{02}\right)^{2}+\cdots+\left(x_{n}-x_{0 n}\right)^{2}\right]^{s} \\
& =\sum_{k_{1}+\cdots+k_{n}=s} \frac{s !}{k_{1} ! \cdots k_{n} !}\left(x_{1}-x_{01}\right)^{2 k_{1}}\left(x_{2}-x_{02}\right)^{2 k_{2}} \cdots\left(x_{n}-x_{0 n}\right)^{2 k_{n}} .
\end{aligned}
$$


We shall call a multi-index "even" if all its components are even, i.e. $q_{i}=2 s_{i}$, $i=1,2, \ldots, n$, and we will write $q=2 s$ for it.

For the even multi-index $q=2 s$ we get from (4.7) that

$$
D^{q}\left(r^{|q|}\right)_{\mid r=0}=D^{q}\left(r^{2|s|}\right)_{\mid r=0}=\frac{|s| ! q !}{(q / 2) !}=\frac{(|q| / 2) ! q !}{(q / 2) !},
$$

where we use the notation $q / 2=s$.

For other powers such that $s \neq|q|, q / 2=s$, we get

$$
D^{q}\left(r^{s}\right)_{\mid r=0}=0 .
$$

In the case when at least one of the indices $q_{i}$ is odd, we get for an arbitrary integer $l \geq 0$,

$$
D^{q}\left(r^{2 l}\right)_{\mid r=0}=0 .
$$

For an arbitrary polynomial $P_{d}(\cdot) \in \operatorname{Pol}(d),(4.8),(4.9)$ and (4.10) give for all even multi-indices $q=2 s$ the following equalities:

$$
D^{q}\left(P_{d}\left(r^{2}\right)\right)_{\mid r=0}=\frac{|s| ! q !}{(q / 2) !} P_{d}^{(s)}(0)
$$

and the equality

$$
D^{q}\left(P_{d}\left(r^{2}\right)\right)_{\mid r=0}=0
$$

for all multi-indices $q$ which are not even.

To simplify the notations let us put $w(x)=\Delta^{k} u(x)$ in (4.6), where according to equalities (4.11) and (4.12), the internal sum becomes

$$
\begin{aligned}
S\left(x_{0}\right) & =\sum_{|q|=p-k} \frac{1}{q !}\left(D^{q} w\right)\left(D^{q} v\right) \\
& =\sum_{\substack{|q|=p-k \\
\text { even } q=2 s}} \frac{(|q| / 2) ! q !}{(q / 2) !} \cdot \frac{1}{q !} w^{(|q| / 2)}(0) D^{q} v\left(x_{0}\right) \\
& =\sum_{\substack{|q|=p-k \\
\text { even } q=2 s}} \frac{(|q| / 2) ! q !}{(q / 2) !} \cdot \frac{1}{q !} w^{(|q| / 2)}(0) D^{q} v\left(x_{0}\right) \\
& =(|q| / 2) ! \cdot w^{(|q| / 2)}(0) \cdot \sum_{\substack{|q|=p-k \\
\text { even } q=2 s}} \frac{1}{(q / 2) !} D^{q} v\left(x_{0}\right) \\
& =(|q| / 2) ! \cdot w^{(|q| / 2)}(0) \Delta^{p-k} v\left(x_{0}\right) .
\end{aligned}
$$

The last quantity is zero for $0 \leq k \leq p-1$, and is $v\left(x_{0}\right)$ for $k=p$. This proves formula (4.5). Q.E.D.

Proof of Proposition 3 follows from Propositions 4 and 5 if we note that the constant $C$ in $(4.1)$ is exactly $l_{p, 2 p}^{n}$.

Now we are prepared to prove the following 
Theorem 2. In the bounded domain $\Omega \subseteq \mathbf{R}^{n}$ let the polyharmonic function $h(x) \in H_{m}(\Omega) \cap C(\bar{\Omega})$. If $R(x)$ denotes the distance from the point $x \in \Omega$ to the boundary $\partial \Omega$ of $\Omega$, then for every integer $p \geq 1$ the sharp inequality

$$
\left|\Delta^{p} h(x)\right| \leq\left(2 / R^{2}(x)\right)^{p} T_{m-1}^{(p)}(1) \cdot\|h\|_{C(\bar{\Omega})}
$$

holds where $T_{m-1}(\cdot)$ is the Chebyshev polynomial of degree $m-1$.

Proof. We shall continue the proof from Theorem 1, using the notations introduced there.

We substitute the same function $h(x)=L_{m-1}\left(r^{2}\right)=T_{m-1}(t)$ for $t=$ $\left(r^{2}-(R / 2)\right) /(R / 2)$.

Following Proposition 4, the left-hand side of (4.1) becomes $l_{p, 2 p}^{n} L_{m-1}^{(p)}(0)$.

As for the right-hand side, the arguments of Proposition 2 apply to show that the $P_{j}^{(p)}(0)$ are alternating with $j=1,2, \ldots, m$.

As a consequence, we get the equality

$$
l_{p, 2 p}^{n} L_{m-1}^{(p)}(0)=\varepsilon l_{p, 2 p}^{n} \cdot \sum_{j=1}^{m}\left|P_{j}^{(p)}(0)\right|,
$$

where $\varepsilon$ is +1 or -1 .

To finish the proof of the inequality we note that the value $\left|L_{m-1}^{(p)}(0)\right|$ is equal to $(2 / R(x))^{p} \cdot T_{m-1}^{(p)}(1)$, since $L_{m-1}^{(p)}(0)=T_{m-1}^{(p)}(-1)$.

The sharpness of (4.13) for every point $x_{0} \in \Omega$ is obtained for the function $L(\cdot)$ by equality (4.14). Q.E.D.

\section{INVERSE THEOREMS FOR THE UNIFORM APPROXIMATION BY POLYHARMONIC FUNCTIONS}

The classical application of Markov inequality (and that of Bernstein in the periodic case) are the so-called inverse theorems, found by S. N. Bernstein in $1912[3,6]$, for the approximation by polynomials in the one-dimensional case. 2.

Here we shall prove the simplest result of this type by using Theorems 1 and

Theorem 3. Let the function $\omega(t)$ (a modulus of continuity type) satisfy the following conditions:

1. $\omega(t)$ is continuous,

2. $\omega(t)$ is monotonely increasing,

3. $\omega(0)=0$,

4. for every $t>0$ the inequality $\omega(2 t) \leq C \omega(t)$ holds with $C=$ const .

5. $\int_{0}^{1} \omega(t) t^{-1} d t<\infty$.

Suppose that for some integer $r \geq 0$ and for a given function $f(x)$ on $\bar{\Omega}$, there exists a series of polyharmonic functions $h_{m}(x) \in H_{m}(\Omega) \cap C(\bar{\Omega})$ which approximate the function $f(x)$ in such a way that for $m=1,2,3, \ldots$ the inequalities

$$
\left|f(x)-h_{m}(x)\right| \leq \frac{A}{m^{2 r}} \omega\left(m^{-1}\right)
$$

hold. Then all derivatives $D^{2 q} f(x)$, with $|q| \leq r$, exist and are continuous in $\Omega$. In particular, $\Delta^{r} f \in C^{(0)}(\Omega)$, and $f \in C^{(r)}(\Omega)$. 
Proof. Let us notice that for every function $h(x) \in H_{m}(\Omega) \cap C(\bar{\Omega})$ we have for every multi-index $q$ with $|q|=r$ the inequality

$$
\left|D^{2 q} h(x)\right| \leq\left|\Delta^{r} h\right|
$$

for every $x \in \Omega$.

We shall denote by $P_{r}$ any of the operators $D^{2 q},|q|=r$, or the operator $\Delta^{r}$. We have, according to Theorem 1 and Theorem 2 , the inequalities

$$
\left|P_{r} h(x)\right| \leq\left(2 / R^{2}(x)\right)^{r}(m-1)^{2 r}\|h\|_{C(\bar{\Omega})}
$$

for every $x \in \Omega$.

For the proof we fix the point $x \in \Omega$.

We shall follow the classical scheme of the proof of Bernstein's inverse theorem [6].

Let us write $f(x)$ in a series:

$$
f(x)=h_{1}(x)+\sum_{j=1}^{\infty}\left[h_{2^{j}}(x)-h_{2^{j-1}}(x)\right] .
$$

Inequality (5.1) gives

$$
\left|h_{2^{k}}(x)-h_{2^{k-1}}(x)\right| \leq 2 \cdot A \cdot 2^{-(k-1) 2 r} \cdot \omega\left(2^{-k+1}\right) .
$$

Hence by inequality (5.2) we get the following

$$
\begin{aligned}
& \left|P_{r} h_{2^{k}}(x)-P_{r} h_{2^{k-1}}(x)\right| \\
& \quad \leq(2 / R(x))^{r} \cdot\left(2^{k}-1\right)^{2 r} A \cdot 2^{-(k-1) 2 r+1} \cdot \omega\left(2^{-k+1}\right) \\
& \quad=(R(x))^{-r} \cdot\left(1-2^{-k}\right)^{2 r} A \cdot C 2^{2 r+1} \cdot \omega\left(2^{-k+1}\right) \leq C_{1}(x) \omega\left(2^{-k}\right) .
\end{aligned}
$$

Here the constant $C_{1}(x)$ does not depend of $k$ but it depends on $x$ through $R(x)$ which is the distance from $x$ to $\partial \Omega$.

Further we choose a positive number $R_{1}$ with $R_{1}<R=R(x)$, and put $C_{1}=\max \left\{C_{1}(y): y \in \bar{B}\left(x ; R_{1}\right)\right\}$ so that if in inequality (5.3) $x$ is replaced by $y$, it would hold for all $y \in B\left(x ; R_{1}\right)$.

Taking into account the monotonicity of $\omega(u)$ we get

$$
\int_{2^{-j}}^{2^{-j+1}} \omega(u) u^{-1} d u \geq \ln 2 \cdot \omega\left(2^{-j}\right) .
$$

This gives

$$
\begin{aligned}
\left|P_{r} f(x)-P_{r} h_{2^{j}}(x)\right| & \leq \sum_{k=j+1}^{\infty}\left|P_{r} h_{2^{k}}(x)-P_{r} h_{2^{k-1}}(x)\right| \\
& \leq C_{1} \int_{0}^{2^{-j}} \omega(u) u^{-1} d u .
\end{aligned}
$$

The last integral goes to zero with $j \rightarrow \infty$ which proves the theorem. Q.E.D.

For an arbitrary function $f(x) \in C^{2 r}(\Omega)$ we shall denote by $\operatorname{Polideg}(f)$ the least integer $s \geq 0$ such that $\Delta^{s} f(x)=0, x \in \Omega$, and we shall call it degree of polyharmonicity. 
Corollary. Let $f(x)$ be a function in $\Omega$. Suppose there exists a sequence of polynomials in $\mathbf{R}^{n}, P_{\nu}(x)$, such that

$$
\left\|f-P_{\nu}\right\| \leq \operatorname{Ad}_{\nu}^{-r} \omega\left(d_{\nu}^{-1}\right)
$$

where $d_{\nu}=\operatorname{Polideg}\left(P_{\nu}\right)$, and $d_{\nu} \rightarrow \infty$ for $\nu \rightarrow \infty$. Then $D^{2 q} f(x)$ exists and is continuous in $\Omega$ for every multi-index $q$ with $|q| \leq r$.

\section{ESTIMATION OF THE MODULUS OF THE INTERMEDIATE DERIVATIVES OF AN ARBITRARY FUNCTION}

For an arbitrary sufficiently smooth function $f(x)$ in $\Omega$, introduce the quantities

$$
M_{0}=\max _{x \in \bar{\Omega}}|f(x)|, \quad M_{p}=\operatorname{essup}_{x \in \bar{\Omega}}\left|\Delta^{p} f(x)\right| .
$$

They will be interesting for us when $M_{0}, M_{p}<\infty$.

Our aim is to estimate the value $\left|\Delta^{j} f(x)\right|, x \in \bar{\Omega}$, through the quantities $M_{0}, M_{p}$.

To do this we need an analogue to the formula of Taylor.

Such an analogue is obtained in [2] through some simple substitutions in the Green formula.

To write it let us recall the fundamental solution of the polyharmonic operator [2]. For the operator $\Delta^{l}$ when the dimension $n$ is odd the fundamental solution is

$$
r_{l}(x)=\frac{r^{2 l-n}}{\gamma_{l-1}} \text { for } l=1,2,3, \ldots .
$$

For even $n=2 m$ we have

$$
\begin{aligned}
& r_{l}(x)=\frac{r^{2 l-n}}{\gamma_{l-1}^{\prime}} \text { for } l=1,2,3, \ldots, m-1, \\
& r_{l}(x)=\frac{-r^{2 l-n} \log r}{\gamma_{l-1}^{\prime}}+\left(c_{l-m} / \gamma_{l-1}^{\prime}\right) \cdot r^{2 l-n} \quad \text { for } l \geq m ;
\end{aligned}
$$

here we denote $r=|x|$. We do not need the exact values of the constants $c, \gamma, \gamma^{\prime}$ which are given in [2] but we shall use only the fact that they satisfy $\Delta^{j} r_{l}(x)=r_{l-j}(x)$ for every integer $j \geq l$.

Denote by $\omega_{n}$ the area of the unit sphere in $\mathbf{R}^{n}$ where $\omega_{n}=2 \pi^{n / 2} / \Gamma(n / 2)$, and put

$$
\Omega_{n}= \begin{cases}1 /(n-2) \omega_{n}, & \text { for } n \neq 2, \\ 1 / \omega_{n}, & \text { for } n=2 .\end{cases}
$$

Here $\Gamma(\cdot)$ denotes the Euler gamma function.

The formula we need is (cf. [2]):

$$
\begin{aligned}
u(x)= & \Omega_{n} \sum_{l=0}^{p-1} \int_{\partial D}\left[\Delta^{l} u(y) \cdot \frac{\partial r_{l+1}(y-x)}{\partial \nu_{y}}\right. \\
& \left.-r_{l+1}(y-x) \cdot \frac{\partial \Delta^{l} u(y)}{\partial \nu_{y}}\right] d \sigma(y) \\
& -\Omega_{n} \int_{D} r_{p}(y-x) \Delta^{p} u(y) d y
\end{aligned}
$$


for every $x \in D$. Here $D$ is a domain in $\mathbf{R}^{n}$ with a sufficiently smooth boundary (e.g. Lyapunov surface, cf. [2]), and $\partial / \partial \nu$ is the inner normal derivative and $d \sigma(x)$ is the element of the intrinsic measure $\sigma$ on $\partial D$. The function $u(x)$ belongs to the class $C^{(2 p)}(\Omega)$.

The following result about the estimate of the intermediate derivatives holds:

Theorem 4. Let for some integer $p \geq 0, \Delta^{p} u(x)$ be a continuous function, including $\Delta^{j} u(x), 0 \leq j \leq p-1$, in the domain $\Omega$. For every positive number $\delta$ denote by $\Omega_{\delta}$ the set $\{x \in \Omega: \operatorname{dist}(x, \partial \Omega)>\delta\}$. If the quantities $M_{0}, M_{p}$, defined by (6.1) are finite then for the intermediate derivatives $\Delta^{j} u(x), j=$ $1,2, \ldots, p-1$, the following inequalities hold:

$$
\max _{x \in \Omega_{\delta}}\left|\Delta^{j} u(x)\right| \leq A^{j} M_{0}+B^{j} M_{p}
$$

where the constants $A^{j}, B^{j}$ depend only on $\delta$.

Proof. By (6.2) we have

$$
u(x)=h_{p}(x)+R_{p}(x), \quad x \in \Omega,
$$

where $\Delta^{p} h_{p}(x)=0, x \in \Omega$.

Suppose that $M_{p}=0$. It follows that $u(x)=h_{p}(x)$. From Theorem 3 we get the inequality

$$
\left|\Delta^{j} h_{p}(x)\right| \leq\left(2 / R^{2}(x)\right)^{j} \cdot T_{p}^{(j)}(1) \cdot M_{0}
$$

for every $x \in \Omega$.

If $M_{p}>0$, we fix some point $x_{0} \in \Omega$, and in formula (6.2) we put $D=B=$ $B\left(x_{0} ; R\right), R=R\left(x_{0}\right)$.

From (6.3) we get

$$
\left|h_{p}(x)\right| \leq|u(x)|+\Omega_{n} M_{p} \int_{B}\left|r_{p}(y-x)\right| d y
$$

for every $x \in B\left(x_{0} ; R\right)$.

We shall estimate the value of the integral

$$
I(x)=\int_{B}\left|r_{p}(y-x)\right| d y
$$

in the point $x_{0}$ for the different cases that arise below.

In the case of odd $n$ we get

$$
I\left(x_{0}\right) \leq \int_{B}\left|r_{p}\left(y-x_{0}\right)\right| d y=\frac{\omega_{n}}{\gamma_{p-1}} \cdot \frac{R^{2 p}}{2 p} .
$$

In the case of even $n=2 m$, for $p=1,2, \ldots, m-1$, we have

$$
I\left(x_{0}\right) \leq \frac{\omega_{n}}{\gamma_{p-1}^{\prime}} \cdot \frac{R^{2 p}}{2 p} .
$$

For $p>m-1$, there are two cases: $R<1$ and $R \geq 1$. For $R<1$ the integral satisfies the inequality

$$
\begin{aligned}
I\left(x_{0}\right) \leq & \left(c_{p-m} \omega_{n} / \gamma_{p-1}^{\prime}\right) R^{2 p}(2 p)^{-1} \\
& -\left(\omega_{n} / \gamma_{p-1}^{\prime}\right)\left[R^{2 p}(2 p)^{-1} \log R-R^{2 p}(2 p)^{-2}\right],
\end{aligned}
$$


and, for $R \geq 1$, the inequality

$$
\begin{aligned}
I\left(x_{0}\right) \leq & \left(c_{p-m} \omega_{n} / \gamma_{p-1}^{\prime}\right) R^{2 p}(2 p)^{-1} \\
& +\left(\omega_{n} / \gamma_{p-1}^{\prime}\right)\left[(2 p)^{-2}+R^{2 p}(2 p)^{-1} \log R-R^{2 p}(2 p)^{-2}\right] .
\end{aligned}
$$

Hence (6.4) becomes

$$
\left|h_{p}(x)\right| \leq M_{0}+M_{p} A_{R, p}
$$

where the constant $A_{R, p}$ is obtained from the above estimates and satisfies $A_{R, p} \rightarrow 0$ for $R \rightarrow 0$, when $p \geq 1$.

From (6.3) through (6.5) we get the inequality

$$
\begin{aligned}
\left|\Delta^{j} u\left(x_{0}\right)\right| \leq & \left(2 / R\left(x_{0}\right)\right)^{j} T_{p-1}^{(j)}(1) \cdot\left[M_{0}+M_{p} A_{R, p}\right] \\
& +\left|\int_{B} \Delta_{x}^{j} r_{p}\left(y-x_{0}\right) \Delta^{p} u(y) d y\right| .
\end{aligned}
$$

Since $\Delta^{j} r_{p}(x)=r_{p-j}(x)$ we get finally the inequality

$$
\begin{aligned}
\left|\Delta^{j} u\left(x_{0}\right)\right| \leq & \left(2 / R\left(x_{0}\right)\right)^{j} T_{p-1}^{(j)}(1) \cdot\left[M_{0}+M_{p} A_{R, p}\right]+M_{p} A_{R, p-j} \\
= & \left(2 / R\left(x_{0}\right)\right)^{j} T_{p-1}^{(j)}(1) \cdot M_{0} \\
& +\left[\left(2 / R\left(x_{0}\right)\right)^{j} T_{p-1}^{(j)}(1) A_{R, p}+A_{R, p-j}\right] \cdot M_{p} .
\end{aligned}
$$

This proves the statement of the theorem by the definition of the set $\Omega_{\delta}$ and the distance function $R(x)$. Q.E.D.

\section{ACKNOWLEDGMENT}

The author would like to thank Professor Zeev Ditzian who read the paper and helped to correct some inaccuracies.

\section{REFERENCES}

1. O. Kounchev, Exact estimation of the Laplacian of a polyharmonic function and approximation through polyharmonic functions, C. R. Acad. Bulgare Sci. 43 (1990), 27-30.

2. N. Aronszajn, T. M. Creese, and L. J. Lipkin, Polyharmonic functions, Clarendon Press, Oxford, 1983.

3. S. N. Bernstein, On the best approximation of continuous functions through polynomials of fixed degree, Collected Works, vol. 1, Publ. Acad. Sci. USSR, 1952, pp. 11-104.

4. S. L. Sobolev, Introduction to the theory of cubature formulas, "Nauka," Moscow, 1974.

5. L. L. Helms, Introduction to potential theory, Wiley, 1963.

6. V. K. Dzyadyk, Introduction to the theory of uniform approximation of functions through polynomials, "Nauka", Moscow, 1977. (Russian)

7. M. Riesz, Formule d'interpolation pour la derivée d'un polynome trigonométrique, Comptes Rendus 158 (1914), 1154.

Bulgarian Academy of Sciences, Institute of Mathematics with Computer Center, Acad. G. Bonchev Str., 8, 1113 Sofia, Bulgaria

E-mail address: banmat@bgearn.bitnet 